Proceedings of the American Control Conference

San Diego, California * June 1999

\title{
Robust Discrete-Time Fuzzy Control of Composite Nonlinear Systems by Tuning Consequent Membership Functions
}

\author{
Wei-Song Lin * and Chih-Hsin Tsai \\ Institute of Electrical Engineering, National Taiwan University, \\ Taiwan, R.O.C \\ Fax: 886-2-23638247 Tel: 886-2-23635251 ext. 413 \\ E-mail weisong@cc.ee.ntu.edu.tw
}

\begin{abstract}
A discrete-time fuzzy controller is proposed to achieve the robust tracking control of composite multivariable nonlinear systems. The control strategy is to decouple the subsystems and to facilitate the robust property by fine-tuning the consequent membership functions of the fuzzy controllers. An on-line tuning mechanism enables the discrete-time fuzzy system to effectively deal with the equivalent uncertainty resulted by function approximation error, disturbance, measurement noise or effect of interconnection among subsystems. Using Lyapunov stability theory, the tracking error residual set of the overall system with the proposed controller has been proved to be uniform ultimate bounded. Simulation results of a two-link robot control demonstrate the effectiveness and robustness of the design.
\end{abstract}

\section{Introduction}

Recently, increasing emphasis has been put on the issues of stability, robustness, and convergence performance of the fuzzy control systems [3]. Using Lyapunov-based learning algorithms, stable adaptive fuzzy controllers have been achieved to ensure stability of the control loop and well-defined behavior of the plant output [4][5][6][7]. Except for single variable systems, the works in [5], [6], and [7] have further investigated stable adaptive multivariable fuzzy control schemes with the fuzzy basis functions either in continuous-time or discrete-time domain. Despite the success of adaptive fuzzy control based on fuzzy basis functions allows the inclusion of a priori knowledge about the plant control. The rules in the form of "IF situation THEN the value of some nonlinear function" are inherent of the plant dynamic rather than the familiar expressions of human expert knowledge. As an improvement, this paper develops a robust fuzzy controller with fuzzy rules in the form of "IF situation THEN the control input". This form of rules not only preserves the superior advantage of the fuzzy systems but also has direct translation from linguistic rules provided by a human expert. Furthermore, since the theory of function approximation using multi-layer fuzzy system has been employed, we come to the second level of endeavor: guaranteeing the robustness of the control against function approximation error, disturbance, measurement noise and effect of interconnection among subsystems.

At this venue, this paper proposes to use two adaptation mechanisms to achieve the goal of robust design. Firstly a parameter adaptation scheme which is widely used in adaptive fuzzy/neural control laws [1][4][5] is adopted to adapt the parameters of the multi-layer fuzzy systems. Then a fine-tuning mechanism of the consequent membership functions of the fuzzy part is proposed and applied to considerably reduce the tracking error residual set. This design obtains robustness in the sense that the fine-tuning mechanism can automatically adapt the controller to react to the effect of equivalent uncertainties of the unknown plant dynamics. The overall adaptation scheme has been proved to be able to guarantee the tracking error residual set to be uniform ultimate bounded.

\section{The control problem}

Consider an $m n^{\text {th }}$-order nonlinear composite discrete-time system in the controllable canonical form

$$
\begin{aligned}
y(k+n) & =f(x(k))+G(x(k)) u(k)+z(x(k), u(k), k) \\
\bar{x}(k) & =x(k)+n_{x}(k) \\
\bar{y}(k) & =y(k)+n_{y}(k)
\end{aligned}
$$

where $y, \bar{y} \in R^{m \prime \prime}$, and $x, \bar{x} \in R^{m n}$ denote the output, measured output, state and measured state vectors, respectively, $x=\left[y_{1}(k), \cdots, y_{m}(k), \quad y_{1}(k+1), \cdots, y_{m}(k+1), \cdots, \quad y_{1}(k+n-1)\right.$, $\left.\cdots, y_{m}(k+n-1)\right]^{T} ; f \in R^{m}, G=$ Block diag $\left[g_{i}(k)\right] \in R^{m}$ are unknown smooth functions satisfying the mild assumption $\left|g_{i}(x(k))\right| \geq g_{i 0}>0, \forall x$, with $g_{i 0}$ being an unknown lower bound. This assumption implies that $g_{i}$ is strictly either positive or negative for all $\boldsymbol{x}$. Hereafter, without loss of generality, it is assumed that $g_{i}$ is strictly positive. $u(k) \in R^{m}$ is the system input; $z(x(k), u(k), k) \in R^{m}$ denotes the unknown interaction among the subsystems and the time-varying disturbance. The exogenous signals $n_{x}$ and $n_{y}$ are assumed to have the properties of standard smoothness and boundedness. Let $y_{M}(k) \in R^{m}$ and $v(k) \in R^{m}$ denote the reference output and input, respectively. The aim of control is to make (1) track a linear reference model of the following form

$$
\begin{aligned}
y_{M}(k+n)=\lambda_{1}^{T} y_{M}(k)+ & \lambda_{2}^{T} y_{M}(k+1)+\cdots+ \\
& \lambda_{n}^{T} y_{M}(k+n-1)+v(k)
\end{aligned}
$$

where the constant matrices $\lambda,=\left[\lambda_{1}, \lambda_{2}, \cdots, \lambda_{m}\right] \in R^{m}$, $j=1, \cdots, n$ are selected so that (2) is asymptotically stable, and the tracking error represented by $e=\left[e_{1}, \cdots, e_{m}\right]^{T}$ with $e_{i}=\left[\bar{y}_{i}(k)-y_{M i}(k), \bar{y}_{i}(k+1)-y_{M i}(k+1), \cdots, \bar{y}_{i}(k+\right.$ $\left.n-1)-y_{M i}(k+n-1)\right]^{T}$ will ultimately be attenuated to small residual tracking error set.

\section{Design of the robust fuzzy controller}

Fig. 1 shows the configuration of the proposed robust fuzzy control system with fine-tuning on the consequent membership functions. Fig. 2 shows the proposed synaptic connection topology of the $t^{\text {th }}$ fuzzy system of the multi-layer fuzzy system. Considering the request of numerical input and output of the fuzzy system, a particular class of fuzzy system with the singleton fuzzified, algebraic product $T$-norm, the sup star compositional operator [4] and the local mean-of-maximum [1] method is used. The basic components of the multi-layer fuzzy system and its fine- 
tuning mechanism will be discussed and formulated in the following paragraph.

Fuzzy Rule Base: A multivariable system can be controlled by the following $N+1$ linguistic rules

$R^{\prime}$ : IF $\bar{x}$ is $A^{j}$ THEN $u$ is $B^{j}$ for $j=1, \ldots, N+I$

The components of the fuzzy set vectors $A^{j}$ and $B^{j}$ are linguistic terms characterized by the fuzzy membership functions

$$
\begin{aligned}
& \mu_{A_{i}^{\prime}}\left(\bar{x}_{k}\right)=\exp \left(-\left(\bar{x}_{k}-m_{k}^{j}\right)^{2} / a_{k}^{j}\right) \quad k=1, \cdots, m n \\
& \mu_{k^{\prime}}\left(u_{i}\right)=\left\{\begin{array}{ll}
\left(1+\left(\left(c_{i}^{\prime}-u_{i}\right) / a_{L_{i}}\right)^{2}\right)^{-1}, & \text { if } u_{i} \leq c_{i}^{j} \\
\left(1+\left(\left(u_{i}-c_{i}^{j}\right) / a_{R i}\right)^{2}\right)^{-1}, & \text { if } u_{i}>c_{i}^{\prime}
\end{array}, \quad i=1, \cdots, m\right.
\end{aligned}
$$$$
\text { and }
$$

where $\left\{a_{k}^{j}, m_{k}^{j}\right\}$ and $\left\{a_{L i}, a_{R i}, c_{i}^{j}\right\}$ are referred to the premise and consequence parameters, respectively.

Rule Credit Assignment: Denote $\omega_{i}^{\prime}>1$ (or $\omega_{i}^{j}<1$ ) as a reward (or a punishment) ffered to the $j^{\text {th }}$ rule, then the consequent membership functions (4) can be reshaped into

$$
\mu_{\bar{B}_{i}^{\prime}}\left(u_{i}\right)= \begin{cases}\left(1+\left(\omega_{i}^{j}\left(c_{i}^{j}-u_{i}\right) / a_{L i}\right)^{2}\right)^{-1}, & \text { if } u_{i} \leq c_{i}^{j} \\ \left(1+\left(\omega_{i}^{j}\left(u_{i}-c_{i}^{j}\right) / a_{R i}\right)^{2}\right)^{-1}, & \text { if } u_{i}>c_{i}^{j}\end{cases}
$$

and the recommendation fuzzy output of each rule becomes

$$
\begin{aligned}
& \omega_{u \prime}^{\prime} \cdot I\left(A^{\prime}\left(\mathbf{x}^{0}\right), \tilde{B}_{i}^{\prime}\left(u_{i}\right)\right) \\
& =\left\{\begin{array}{cc}
\omega_{v}^{\prime} \cdot A^{\prime}\left(\mathbf{x}^{0}\right), & \text { foru } \\
0, & \text { otherwise }
\end{array},\right.
\end{aligned}
$$

where "." is the multiplication operation, $I$ is the implication function [4], $\mu^{\prime}(\bar{x})=\prod_{k=1}^{m n} \mu_{A_{i}^{\prime}}\left(\bar{x}_{k}\right)$ denotes the given input and the matching degree, respectively, $\widetilde{c}_{i}^{j}$ denotes the location of the singleton implication fuzzy set and is defined as

$\widetilde{c}_{i}^{\prime}=$ the centroid of the set $\left\{u_{i}: \mu_{\tilde{B}_{i}^{\prime}}\left(u_{i}\right) \geq \mu^{j}(\bar{x})\right\}$

Using (5), (7) can be resolved into

$$
\tilde{c}_{i}^{\prime}=c_{i}^{j}-a_{L R i} \mu^{j} \sqrt{\left(\mu^{j}\right)^{-1}-1} / \omega_{i}^{j}
$$

where $a_{L R i}=\left(a_{U}-a_{R i}\right) / 2$.

Fine-tuning mechanism: Physically, the parameter $a_{L R i}$ represents the difference between the left and right spreads of the consequent membership functions. In traditional fuzzy logic control system, $a_{L i}$ is set to be equivalent to $a_{R i}$ or the consequent membership is just in singleton form [4]. In this paper, this term is employed as a robust control component and a robust adaptive law for it is proposed in the next section.

Analytical formulation of the multi-layer fuzzy system: Using the center average defuzzification, the output response of the fuzzy controller is

$$
u_{i}(k)=F_{i}\left(\bar{x}, \omega_{i}^{j}, a_{L R i}\right)=\frac{\sum_{j=1}^{N+1} \omega_{i}^{j} \cdot \mu^{j} \cdot \tilde{c}_{i}^{j}}{\sum_{j=1}^{N+1} \omega_{i}^{j} \cdot \mu^{j}}
$$

In the rule base, the $(N+I)^{\text {th }}$ rule is chosen to be of TakagiSugeno type and its consequent fuzzy set vector $B^{N+1}$ is singleton with support represented as the form of the synthesis input

$$
c^{\prime}(k)=\lambda_{1}^{T} \bar{y}(k)+\lambda_{2}^{T} \bar{y}(k+1)+\cdots+\lambda_{n}^{T} \bar{y}(k+n-1)+v(k) \quad(10)
$$

On the other hand, the curvature control parameter, $a_{k}^{N+1}$, of its antecedent membership functions is assumed to approach to infinity so that this rule will be fired whatever $x$ is. The credit assignment takes place in rules $R^{j}, j=1, \ldots, N$, but assigned to be 1 for $R^{N+1}$. Accordingly, using (8) and (10), the analytical formulation of the multi-layer fuzzy system in equation (9) resolves into

$$
u=\hat{G}^{-1}\left(-\hat{f}+c^{\prime}-a_{L R}^{T} \mu_{L R}\right)
$$

where, $\hat{G}=B \operatorname{lock} \operatorname{diag}\left[\omega_{i}^{T}(k+1) \mu^{\prime}(k)\right]_{m \times m}, \omega_{i}(k+1)$ and $\mu^{\prime}(k)$ are $(N+I) \times 1$ column vectors composed of $\omega_{i}^{j}(k+1)$ and $\mu^{i}(k), \hat{f}=\left[\beta_{i}^{T}(k+1) \mu(k)\right]_{m}, \beta_{i}(k+1)$ and $\mu(k)$ are $N \times 1$ column vectors composed of $\omega_{i}^{j}(k+1) c_{i}^{j}(k+1)$ and $\mu^{j}(k), a_{L R}(k+1) \in R^{m}$, and $\mu_{L R}=\sum_{j=1}^{N} \mu^{j} \sqrt{\left(\mu^{j}\right)^{-1}-1} \in R$. When $\omega_{i}=0$ the control law (11) is not well-defined. To avoid this situation, we project $\theta=\left[\beta_{1}^{T}, \omega_{1}^{T}, \cdots, \beta_{m}^{T}, \omega_{m}^{T}\right]^{T}$ into an estimated feasible region $M_{\theta}=\left\{\theta:\left|\beta_{i}\right| \leq \beta_{i, \text { Max }}, \quad 0<\omega_{i}\right.$ $\left.\leq \omega_{i, \operatorname{Max}}\right\}$ by properly adapting the parameter values.

\section{Learning algorithm and performance analysis}

Let $\theta^{*}=\left[\beta_{1}^{* T}, \omega_{1}^{* T}, \cdots, \beta_{m}^{* T}, \omega_{m}^{* T}\right]^{T}$ and define the parameters of the best function approximation to be

$$
\begin{aligned}
& \beta_{i}^{*}=\arg \min _{\beta_{i} \in M_{i}}\left[\sup \left|f_{i}-\beta_{i}^{T} \mu\right|\right] \\
& \omega_{i}^{*}=\arg \min _{\omega, \in M_{0}}\left[\sup \left|g_{i}-\omega_{i}^{T} \mu^{\prime}\right|\right]
\end{aligned}
$$

Then (1) can be rewritten in terms of the measured output $\bar{y}(k)$ and expressed as

$$
\begin{aligned}
\bar{y}(k+n) & =f(x)+G(x) u+z(x, u, k)+n_{y}(k+n) \\
& =\hat{f}^{*}(\bar{x})+\zeta_{f}+\left(\hat{G}^{*}(\bar{x})+\zeta_{z}\right) u+z(x, u, k)+n_{y}(k+n)
\end{aligned}
$$

where $\hat{f}^{*}(\bar{x})=\left[\beta_{i}^{{ }^{*}} \mu(\bar{x})\right]_{m}, \quad \hat{G}^{*}(\bar{x})=$ Block diag $\left[\omega_{i}^{* T} \mu^{\prime}(\bar{x})\right]_{m \times m}$ and

$$
\begin{aligned}
& \zeta_{f}=f(x)-\left[\beta_{i}^{* r} \mu(x)\right]_{m}-\left[\beta_{i}^{* r} \Delta \mu\left(x, n_{x}\right)\right]_{m} \\
& \zeta_{g}=\text { Block diag }\left[g_{i}(x)-\omega_{i}^{\cdot r} \mu^{\prime}(x)-\omega_{i}^{* r} \Delta \mu^{\prime}\left(x, n_{x}\right)\right]_{m \times m}
\end{aligned}
$$
and

$$
\begin{aligned}
& \Delta \mu\left(x, n_{x}\right)=\mu(\bar{x})-\mu(x) \\
& \Delta \mu^{\prime}\left(x, n_{x}\right)=\mu^{\prime}(\bar{x})-\mu^{\prime}(x)
\end{aligned}
$$

are measure of sensitivity of the nominal model $z(x, u, k) \equiv n_{x} \equiv 0$ with respect to the measurement noise $n_{x}$. By (11) and (2), subtracting $\hat{G} u$ and adding $-\hat{f}+c^{\prime}-a_{L R}^{T} \mu_{L R}$ to the right hand side of (13), we obtain the following error equation

$$
\begin{aligned}
& \bar{y}(k+n)-y_{M}(k+n)=-\lambda_{1}^{T}\left(\bar{y}(k)-y_{M}(k)\right)-\cdots-\lambda_{n}^{T}(\bar{y}(k+n-1)- \\
& \text { or } \begin{aligned}
&\left.y_{M}(k+n-1)\right)-\widetilde{f}(\bar{x})-\widetilde{G}(\bar{x}) u+\zeta-a_{L R}^{T} \mu_{L R} \\
& e(k+1)= A e(k)-B \phi^{T}(k) \widetilde{\theta}(k+1)- \\
& B a_{L R}(k+1) \mu_{L R}(\bar{x}(k))+B \zeta(k)
\end{aligned}
\end{aligned}
$$


where

$\zeta=\zeta_{f}+\zeta_{g} u+z(x, u, k)+n_{\nu}(k+n)$,

$\widetilde{f}(\bar{x})=\left[\left(\beta_{i}^{T}(k+1)-\beta_{i}^{* T}\right) \mu(\bar{x})\right]_{m}$,

$\widetilde{G}(\bar{x})=$ Block $\operatorname{diag}\left[\left(\omega_{i}^{T}(k+1)-\omega_{i}^{* T}\right) \mu^{\prime}(\bar{x})\right]_{m \times m}$,

$A=$ Block $\operatorname{diag}\left[A_{1}\right]_{m \times n}, B=$ Block diag $\left[b_{1}\right]_{m \times 1}$, and $\tilde{\theta}(k+1)=\cdot$

$\theta(k+1)-\theta^{*}$ denotes the parameter estimation error and

$$
A_{i}=\left[\begin{array}{ccccc}
0 & 1 & 0 & \cdots & 0 \\
0 & 0 & 1 & \cdots & 0 \\
\vdots & \vdots & \vdots & \ddots & \vdots \\
0 & 0 & 0 & \cdots & 1 \\
-\lambda_{i 1} & -\lambda_{i 2} & -\lambda_{i 3} & \cdots & -\lambda_{i n}
\end{array}\right], \quad b_{i}=\left[\begin{array}{c}
0 \\
0 \\
\vdots \\
0 \\
1
\end{array}\right], \phi=\left[\begin{array}{ccc}
\mu & \cdots & 0 \\
\mu^{\prime} u_{1} & \cdots & 0 \\
\vdots & \ddots & \vdots \\
0 & \cdots & \mu \\
0 & \cdots & \mu^{\prime} u_{m}
\end{array}\right]
$$

Assumption 1: There exists $\bar{\zeta}$ such that

$$
\bar{\zeta}^{2}=\max _{k}\|\zeta(k)\|^{2}
$$

In the following paragraph, the parameter adaptation scheme and the fine-tuning mechanism of the consequent membership functions are proposed.

Parameter adaptation scheme: Inspired by previous work in discrete-time adaptive control [2], we first propose the parameter adaptation law for $a_{L R} \equiv 0$ as

$$
\theta(k+1)=\theta(k)+\Gamma_{1} \phi(k) B^{T} P e(k+1)-\sigma_{1}\left(\theta(k)-\theta_{0}\right)(20)
$$

where $\Gamma_{1}$ is a diagonal matrix with positive diagonal elements, $P=$ Block diag $\left[P_{1}, \cdots, P_{n}\right]$ is a symmetric positive-definite matrix satisfying the Lyapunov equation $\mathrm{A}^{\top} P+P A=-Q$, with the design parameters $Q>0$, and $0<\sigma_{1}<1$ usually referred to as leakage. Note that the adaptive control law depends on the tracking error $e(k+1)$, which is not available at discrete-time $k$. Using a priori estimate $\hat{e}(k+1)$ of $e(k+1)$ can solve this difficulty, and this $\hat{e}(k+1)$ can be calculated from available information at discrete-time $k$. Following the same argument of [2], the a priori estimate is suggested to be $\hat{e}(k+1)=$ $\left[I+B \phi^{T} \Gamma_{1}^{T} \phi B^{T} P\right]^{-1} e(k)$.

Fine-tuning mechanism for consequent membership functions: The parameter $a_{L R}$ is chosen as $a_{L R}(\vartheta)=\varphi^{\prime}(e) \vartheta$, where $\vartheta \in R^{m}$ is an auxiliary adjustable parameter and $\varphi^{\prime}=\operatorname{Block} \operatorname{diag}\left[\varphi_{i}^{\prime}\right]_{m \times m}$, $\varphi_{i}^{\prime}=\tanh \left(\frac{b_{i}^{T} P_{i} e_{i}(k+1) \mu_{L R}}{\epsilon}\right), \mathcal{E}$ is a small positive constant. Using the following assumption:

Assumption 2: There exists the smallest non-negative parameter vector $\vartheta^{*} \geq 0$ such that for all $\bar{x} \in \Re^{m n}$ and $k \geq 1$

$$
|\zeta| \leq \vartheta^{*} \mu_{L R}(\bar{x}(k))
$$

Let $M_{\vartheta}=\left\{\vartheta: 0<\vartheta_{i} \leq \vartheta_{i, \text { Mar }}\right\}$ be the bound of $\vartheta$. We propose the following fine-tuning mechanism for the consequent membership functions

$$
\vartheta(k+1)=\vartheta(k)+\Gamma_{2} \varphi B^{T} P e(k+1)-\sigma_{2}\left(\vartheta(k)-\vartheta_{0}\right)
$$

where $\Gamma_{2}$ is a diagonal matrix with positive diagonal elements, $\varphi=\varphi^{\prime} \mu_{L R}$, and $0<\sigma_{2}<1$ is chosen small but positive constant.

Theorem 1: Suppose that the desired trajectory $y_{M}(k)$ and the initial condition be bounded in a compact set $S$. Consider the nonlinear composite system (1), with the multi-layer fuzzy controller (11) and the parameter adaptation provided by either Algorithm 1) using the adaptation law (20) without tuning consequent membership functions i.e., $a_{L R} \equiv 0$; or

Algorithm 2) using the adaptation law (20) with the fine-tuning mechanism (22).

Then, the tracking error $e(k)$ and the parameters $\theta$ and $\vartheta$ are UUB, and the practical bounds are given by

$$
\Omega=\left\{e(k), \theta(k): \frac{1}{2} \lambda_{\min }(Q)\|e(k)\|^{2}+\sigma_{1} \lambda_{\min }\left(\Gamma_{1}^{-1}\right)\|\tilde{\theta}(k)\|^{2} \leq D_{1}\right\}
$$

where

$$
\begin{gathered}
D_{1}=\sigma_{1} \lambda_{\text {max }}\left(\Gamma_{1}^{-1}\right)\left\|\left(\theta^{*}-\theta_{0}\right)\right\|^{2}+\lambda_{\text {max }}(P) \bar{\zeta}^{2}(1+ \\
\left.2 \frac{\lambda_{\text {max }}(P)}{\lambda_{\text {min }}(Q)}\|A\|^{2}\right)
\end{gathered}
$$

for the case of algorithm 1 ; and

$$
\begin{gathered}
\Omega=\left\{e(k), \theta(k), \vartheta(k): \lambda_{\operatorname{mix}}(Q)\|e(k)\|^{2}+\sigma_{1} \lambda_{\text {min }}\left(\Gamma_{1}^{-1}\right)\|\tilde{\theta}(k)\|^{2}+\right. \\
\left.\sigma_{2} \lambda_{\min }\left(\Gamma_{2}^{-1}\right)\|\widetilde{\vartheta}(k)\|^{2} \leq D_{2}\right\}
\end{gathered}
$$

where

$$
\begin{aligned}
& D_{2}=\sigma_{1} \lambda_{\max }\left(\Gamma_{1}^{-1}\right)\left\|\left(\theta^{*}-\theta_{0}\right)\right\|^{2}+\sigma_{2} \lambda_{\max }\left(\Gamma_{2}^{-1}\right)\left\|\left(\vartheta^{*}-\vartheta_{0}\right)\right\|^{2} \\
& +2 \sum_{i=1}^{m} \vartheta_{i}^{M} \kappa \varepsilon
\end{aligned}
$$

for the case of algorithm 2, provided the Assumption 1 and 2 hold for algorithm 1 and 2, respectively.

Proof. Omitted due to limitation of space.

Remark 1: In algorithm 1, which shuts down the fine-tuning mechanism (i.e., $a_{L R} \equiv 0$ ), the size of tracking error residual set will depend on $\bar{\zeta}^{2}$ which varies with the control effort, effect of interconnection among subsystems, measurement noise and disturbance. But this does not happen in algorithm 2 that obtains some degree of "adaptability" by the fine-tuning mechanism, $a_{L R} \mu_{L R}$, which deals with different values of $\zeta$.

\section{Simulation}

The equations of motion of the arm can be expressed in matrix form as follows:

$$
\begin{aligned}
& {\left[\begin{array}{c}
\left(m_{1}+m_{2}\right) r_{1}^{2}+m_{2} r_{2}^{2}+2 m_{2} r_{1} r_{2} c_{2}+J_{1} m_{2} r_{2}^{2}+m_{2} r_{1} r_{2} c_{2} \\
m_{2} r_{2}^{2}+m_{2} r_{1} r_{2} c_{2} \quad m_{2} r_{2}^{2}+J_{2}
\end{array}\right]\left[\begin{array}{l}
\ddot{q}_{1} \\
\ddot{q}_{2}
\end{array}\right]+} \\
& {\left[\begin{array}{c}
-m_{2} r_{1} r_{2} s_{2} \dot{q}_{1}\left(\dot{q}_{1}+\dot{q}_{2}\right) \\
m_{2} r_{1} r_{2} s_{2} \dot{q}_{2}^{2}
\end{array}\right]+\left[\begin{array}{c}
\left(\left(m_{1}+m_{2}\right) l_{1} c_{2}+m_{2} l_{2} c_{12}\right) g \\
\left(m_{2} l_{2} c_{12}\right) g
\end{array}\right]=\left[\begin{array}{l}
u_{1} \\
u_{2}
\end{array}\right]+\left[\begin{array}{l}
d_{1} \\
d_{2}
\end{array}\right],}
\end{aligned}
$$

where $m_{1}, m_{2}, J_{1}, J_{2}, r_{1}=0.5 l_{1}$, and $r_{2}=0.5 l_{2}$ are the mass, the moment of inertia, the half length of link 1 and 2 , and $c_{1} \equiv \cos \left(q_{1}\right), \quad s_{12} \equiv \sin \left(q_{1}+q_{2}\right)$, etc. The combined effects of friction and the external torque disturbance are 


$$
\begin{aligned}
& d_{1}=2.0 \sin \left(\dot{q}_{1}\right)+2.5 \sin \left(\dot{q}_{2}\right)+0.5 \sin (t) \\
& d_{2}=5.0 \sin \left(\dot{q}_{1}\right)+4.0 \sin \left(\dot{q}_{2}\right)+0.4 \sin (t)
\end{aligned}
$$

In the control experiments described below, the kinematics and inertial parameters of the arm are chosen as $m_{1}=0.5 \mathrm{~kg}$, $m_{2}=6.25 \mathrm{~kg}, \quad J_{1}=J_{2}=5.0 \mathrm{~kg} \cdot \mathrm{m}, \quad l_{1}=2.0 \mathrm{~m}, l_{2}=1.6 \mathrm{~m}$, respectively. The excessive ratio between $m_{1}$ and $m_{2}$ is to emphasize the load effect. As shown in Fig. 3, the trajectory to be followed by the $i^{\text {th }}$ joint is given by two decoupled linear systems as

$$
q_{\mathrm{A} i}(k+2)=\lambda_{1,1} q_{\mathrm{Ait}}(k)+\lambda_{i 2} q_{\mathrm{iti}}(k+1)+v_{i}(k), \quad i=1,2
$$

The model parameters are chosen as follows: $\lambda_{11}=\lambda_{12}=-1.0$, $\lambda_{21}=\lambda_{22}=-1.0$ and the driving inputs to the reference models are sinusoidal functions $v_{1}(k)=\pi \sin (0.8 \pi k)$, and $\nu_{2}(k)=1.5 \pi \cos (\pi k)$. The initial conditions are set to be $q_{1}(0)=-1.57 \mathrm{rad}, \quad q_{2}(0)=0 \mathrm{rad}, \quad \dot{q}_{1}(0)=\mathrm{rad} / \mathrm{sec}, \quad$ and $\dot{q}_{2}(0)=0 \mathrm{rad} / \mathrm{sec}$, The simulations have been done by using a fourth order Runge-Kutta algorithm with an integration step of $0.005 \mathrm{sec}$., and the sampling interval for the controller is set to $0.02 \mathrm{sec}$. The design parameters are given by $Q=10 I_{4 \times 4}, \Gamma_{1}=$ Block diag $\left[5.0 I_{81 \times 81}, 10^{-4} I_{81 \times 81}, 10.0 I_{81 \times 81}, 10^{-4} I_{81 \times 81}\right], \Gamma_{1}=$ Block diag [0.01, 0.02], $\sigma_{1}=\sigma_{2}=10^{-4}$, and $\varepsilon=0.1$. The elements in $\beta_{i}$ and $\omega_{i}$ are chosen randomly in the interval $(-10$, $10)$ and $(0.01,2)$, respectively. The membership functions of state $q_{1}(k-1), q_{1}(k), q_{2}(k-1)$, and $q_{2}(k)$ (represented by generic variable $x_{k}$ ) for $M=3^{4}=81$ regular rule partitions are defined as $\{\mathrm{NB}, \mathrm{ZE}, \mathrm{PB}\}$ where NB: $A_{k}^{j}\left(x_{k}\right)=\exp \left(-4\left(x_{k}+1.8\right)^{2}\right), \mathrm{ZE}$ : $A_{k}^{\prime}\left(x_{k}\right)=\exp \left(-4 x_{k}^{2}\right)$, PB: $A_{k}^{j}\left(x_{k}\right)=\exp \left(-4\left(x_{k}-1.8\right)^{2}\right)$.

\section{A. Tracking performance using algorithms 1 and 2}

Fig. 4 shows the tracking performance using algorithm 1 (i.e. without the fine-tuning mechanism). Fig. 5 shows the tracking performance of using algorithm 2 (i.e. with the fine-tuning mechanism). The dashed and solid lines correspond to the desired and the controlled angular trajectories of the robot-arm, respectively.

\section{B. Tracking with measurement noise}

This simulation investigates the tracking control of the multilayer fuzzy system with algorithm 2 when measurement noises being involved. The noise are assumed to be white with uniform distribution within $[-0.01,0.01](\mathrm{rad})$. The effects of noise of different sensors are assumed to be independent of each other. The results of control are depicted in Fig. 6.

\section{Tracking under re-initialization error and load variation}

In this simulation, the performance of the proposed controller is investigated for re-initialization error and load variation. The reinitialization error is set to within an admissible deviation level, $q_{1}(0)-q_{M / 1}(0)=-0.05$, and $q_{2}(0)-q_{M 2}(0)=0.05$, and $m_{2}$ of the robot is changed from 6.25 to $10.0 \mathrm{~kg}$ at the begging of the third second. The simulation results (plotted in Fig. 7) show that the design is robust to initial state error and load variation.

\section{Conclusion}

A novel multi-layer fuzzy controller for robust discrete-time tracking control of composite multivariable nonlinear systems has been proposed. The fuzzy system with rule credit assignment and fine-tuning mechanism serves two purposes. First, unlike previous works of adaptive fuzzy controller, the form of rules preserves the superior advantage of the fuzzy system. Second, by on-line tuning the consequent membership functions, the multi-layer fuzzy controller achieves some degree of robust properties and considerably reduces the tracking error residual set. The tuning mechanism can automatically adapt the fuzzy controller to react to the effect of the equivalent uncertainty in unknown plant dynamics. The performance of the algorithms with and without tuning consequent membership function has been investigated and compared by an example of a two-link robot control, and the overall design has been proved theoretically to be able to guarantee uniform ultimate boundedness.

\section{Acknowledgement}

The financial support for this research from the National Science Council of Taiwan, R.O.C. under NSC 88-2212-E-002-070 is gratefully acknowledged.

\section{References}

[1] H. R. Berenji and P. S. Khefkar, "Learning and tuning fuzzy logic controllers through reinforcements," IEEE Trans. Neural Networks, vol. 3, pp. 725-740, 1992.

[2] M. Kamoun, A. Titli and M.B.A. Kamoun, "Robust decentralized model reference adaptive control of discretetime interconnected systems," Int. J. Systems Sci, vol. 23, no. 11, pp. 1841-1856, 1992.

[3] T. A. Johansen, "Fuzzy model based control: Stability, robustness, and performance issues," IEEE Trans. Fuzzy Systems, vol. 2, pp.221-234, 1994.

[4] L. X. Wang, Adaptive Fuzzy Systems and Control: Design and Stability Analysis NJ. Prentice-Hall, 1994.

[5] R. Ordonez, J. T. Spooner and K. M. Passino, "Stable multiinput multi-output adaptive fuzzy control," Proceedings of the IEEE Conference on Decision and Control, vol. 1, pp.610-615, 1996.

[6] T. K. Yin and C.S.G. Lee, "Fuzzy model-reference adaptive control," IEEE Trans. Systems, Man, and Cybernet., vol. 25, no. 12, pp.1606-1615, 1995.

[7] S. Jagannathan, "Adaptive fuzzy logic control of feedback linearizable discrete-time nonlinears systems," Proceedings of the 1996 IEEE International Symposium on intelligent Control, vol. 1, pp.133-138, 1996.

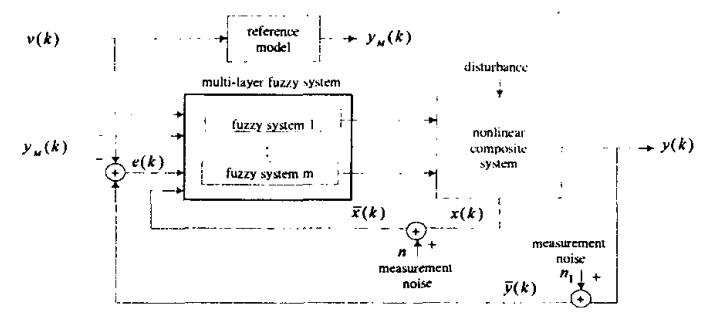

Fig. 1. Configuration of the proposed robust fuzzy control system. 


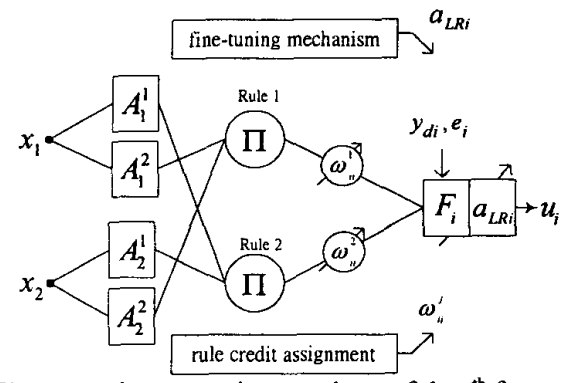

Fig. 2. The synaptic connection topology of the $t^{\text {th }}$ fuzzy system.

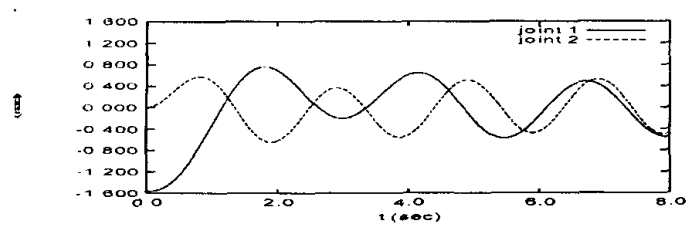

Fig. 3. Reference output of joint 1 and 2.
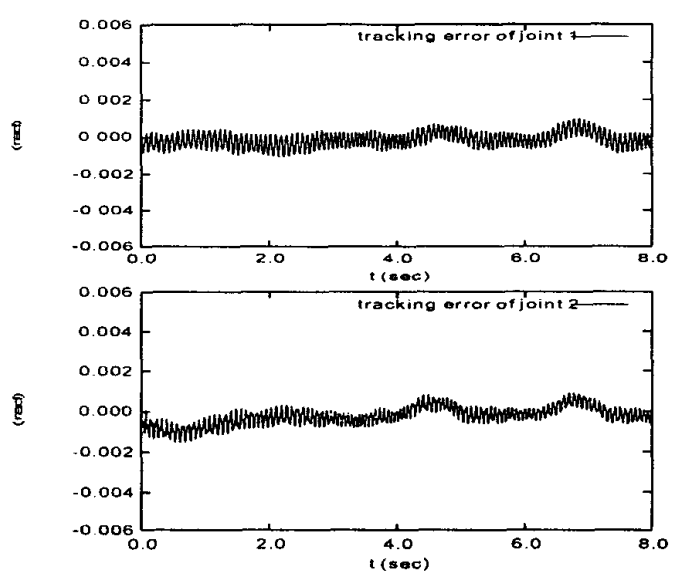

Fig. 4. Tracking performance of using algorithm 1 (without finetuning mechanism).
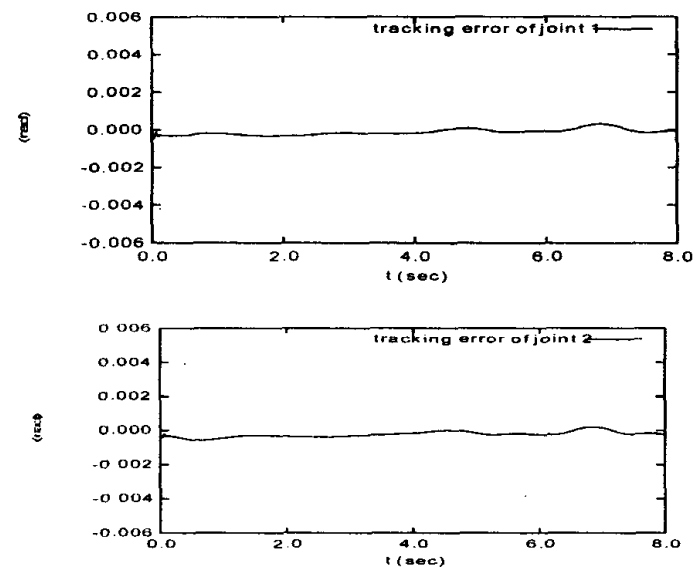

Fig. 5. Tracking performance of using algorithm 2 (with finetuning mechanism).
妾

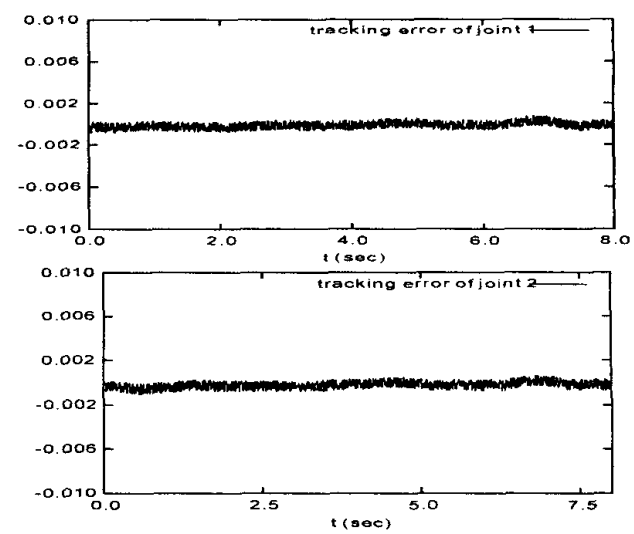

Fig. 6. Tracking performance of using algorithm 2 considering measurement noise.

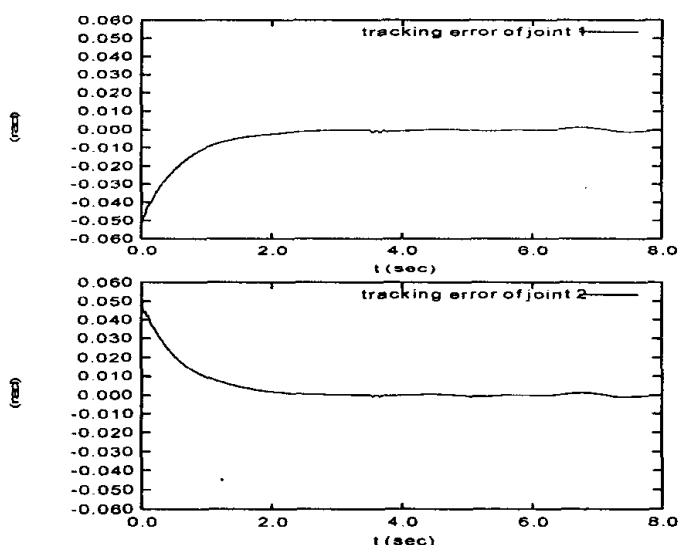

Fig. 7. Tracking performance of using algorithm 2 with reinitialization error and a load change. 